\title{
Real-time detection of influenza A virus using semiconductor nanophotonics
}

\author{
Dominic Lepage, Alvaro Jiménez, Jacques Beauvais and Jan J Dubowski
}

Modern miniaturization and the digitalization of characterization instruments greatly facilitate the diffusion of technological advances in new fields and generate innovative applications. The concept of a portable, inexpensive and semi-automated biosensing platform, or lab-on-a-chip, is a vision shared by many researchers and venture industries. Under this scope, we present a semiconductor monolithic integration approach to conduct surface plasmon resonance studies. This technology is already commonly used for biochemical characterization in pharmaceutical industries, but we have reduced the technological platform to a few nanometers in scale on a semiconductor chip. We evaluate the signal quality of this nanophotonic device using hyperspectral-imaging technology, and we compare its performance with that of a standard prism-based commercial system. Two standard biochemical agents are employed for this characterization study: bovine serum albumin and inactivated influenza A virus. Time resolutions of data acquisition varying between 360 and $2.2 \mathrm{~s}$ are presented, yielding $2.7 \times 10^{-5}-1.5 \times 10^{-6}$ RIU resolutions, respectively.

Light: Science \& Applications (2013) 2, e62; doi:10.1038/lsa.2013.18; published online 12 April 2013

Keywords: GaAs/AIGaAs quantum well device; hyperspectral imaging technology; influenza A virus; integrated surface plasmon resonance biosensor

\section{INTRODUCTION}

Recent advancements in molecular detection methods, microelectronics and digital computing have enabled the development of many new tools and applications in biomedical technology. Examples of this technological democratization include the novel telesurgery procedures, ${ }^{1}$ the advent of electronic personal glucometers or the more recent coming of mobile phone microscopy. ${ }^{2}$ However, the delocalization of arbitrary biochemical analyses is still a challenge today, where nanotechnogy could present solutions for the development of micro total analysis systems ( $\mu$ TAS) potentially capable of portable biodiagnostics. ${ }^{3}$ We present here the results of an effort towards such a solution through a wholly integrated semiconductor-based surface plasmon resonance (SPR) nanometric platform.

SPR is a well-established optical phenomenon where an electromagnetic (EM) beam of a specific energy and incident wavevector (angle) can induce a resonant group oscillation within the surface electrons of a metal-dielectric interface. ${ }^{4}$ The resulting EM field is evanescent in nature, with typical confinement of $200 \mathrm{~nm}$ for visible light. Therefore, the coupling conditions of this resonance effect are very much dictated by the surface conditions within the evanescent field and can consequently be employed for many dynamic biochemistry studies. ${ }^{5}$ The SPR biocharacterization platform presents many advantages over most other methods; its range of application is very broad, especially for unspecific binding studies, ${ }^{5}$ the method can offer very high sensitivities to subtle surface refractive index changes ${ }^{6}$ and even enable subwavelength surface imagery. ${ }^{7,8}$ However, the technological platform for carrying out even basic SPR tracking measurements is relatively bulky, expensive and requires highly qualified personnel to operate and interpret the results. Thus, while SPR instruments are commercially available, their daily use remains confined within specific research fields for both industry and academia. The cross-hybridization of SPR technology with other diagnostic platforms, as would be fundamentally required by any $\mu \mathrm{TAS},{ }^{3}$ has thus far been relatively impractical.

We envision an effective introduction of the SPR technology as a $\mu$ TAS transducer, which would ideally involve the integration of the transduction method into a platform compatible with existing semiconductor and microelectronic systems. The innovative combination of these items would enable a great diversification of the SPR technique for biosensing applications. However, successfully achieving this integration requires advanced understanding of semiconductorSPR events, as the system's complexity is translated away from the targeted users to the system's designers.

We expose how it is possible to monolithically integrate an SPR platform with embedded semiconductor light emitters, use it to carry out biochemical analyses and detect a specific strain of the influenza A virus (IAV). Other lab-on-chip technologies miniaturizing the SPR method are limited either by the physical dimensions of the prism used for near-field coupling, or by the nature of the light sources, imposing macroscopic limitations on the SPR device geometry. With our

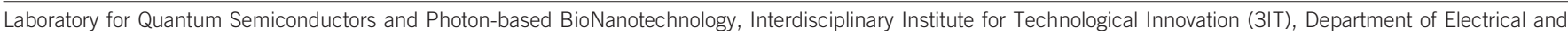
Computer Engineering, Université de Sherbrooke, Sherbrooke, QC J1K 2R1, Canada

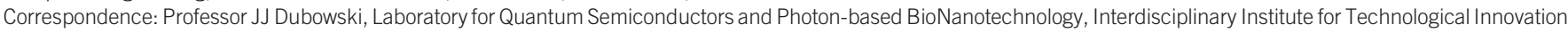
(3IT), Department of Electrical and Computer Engineering, Université de Sherbrooke, Sherbrooke, QC J1K 2R1, Canada 
approach, the light source-transducer distance has been brought to the ultimate minimum. The consequence is a broadband and uncollimated biosensing platform. We show how this type of EM emissions can be employed to track the entire plasmonic dispersion relation. This original method, enabled through hyperspectral imaging, has never been taken advantage of. Recently, we have reported on the in-depth analysis of the static response of a quantum well (QW) semiconductor SPR device. ${ }^{9}$ Here, the sensitivity threshold of our QW-SPR chip is studied using the dynamic physisorption of bovine serum albumin (BSA) and compared between three different SPR methods: a standard prism-based approach and two hyperspectral processes. Following our research interests in methods for detecting IAV immobilized on semiconductor ${ }^{10}$ and gold ${ }^{11}$ surfaces, we examine a series of procedures for the detection of inactivated IAV-H3N2 using QW-SPR biochips.

\section{MATERIALS AND METHODS}

The SPR effect is based on the coupling of electron surface fluctuations with an incoming EM field at a metal-dielectric interface. The resonance occurs following a specific dispersion relation, ${ }^{4}$ where the relationship between the coupling energy $E$ and wavevector $\mathbf{k}(\theta)=\mathbf{k}_{x} \hat{x}+\mathbf{k}_{y} \hat{y}+\mathbf{k}_{z} \hat{z}$, of the resulting plasmonic quasi-particles will be closely related to the dielectric nature of the surface vicinity ( $<200 \mathrm{~nm}$ for visible light). Traditionally, either $E$ or $\mathbf{k}$ is fixed using a laser to pursue dynamic resonance tracking of surficial events. However, the SPR phenomenon is a multidimensional occurrence in energies and planar wavevectors at a given boundary. ${ }^{12,13}$ This phenomenon is illustrated in Figure 1a, where a continuum of surface plasmon (SP) resonances can be induced along the $E(\mathbf{k})$ dimensions. Studying resonances as a function of those variables would yield spectral and directional information on the local dielectric values of the neighbouring nano-objects. The full dispersion of the SPR is given in $I\left(E, \mathbf{k}_{x}, \mathbf{k}_{y}\right)$, as illustrated in Figure $1 \mathrm{~b}$, where the resonance phenomena occurs outside the free propagating light-cone.

In the case of monolithic integration of the SPR platform with an embedded semiconductor light emitter, various designs can be employed to different ends. To better understand the nanophotonic interactions within the structure, an analytical calculation tool was required to predict the far field outputs and to optimize all the existing variables involved in the fabrication of the QW-SPR device. An efficient method was developed from Glytsis and Gaylord's ${ }^{14}$ rigorous coupled wave analysis, which was adapted in a tensor version to take into account multiple variables concurrently. ${ }^{15,16}$ This approach has been proven to be very effective for calculating most photonic interactions of the layered device. Thus, it became possible to predict the output of any QW-SPR architecture and optimize it for specific biochemical testings. ${ }^{16}$

A cross-section of the ensuing device is presented in Figure 2. The fabrication of the prototypes presented here first involved the deposition of $722 \mathrm{~nm}$ of $\mathrm{SiO}_{2}$ atop a single GaAs-AlGaAs QW microstructure emitting at $E=1.42 \mathrm{eV}$. A $20 \mathrm{~nm}$-thick continuous Au layer, corrugated by a one-dimensional, $20 \mathrm{~nm}$-thick Au grating of periodicity $P=669 \mathrm{~nm}$ and line/groove ratio of 1.35 , was fabricated on top of the $\mathrm{SiO}_{2}$ layer. Using tensorial rigorous coupled wave analysis, the various thicknesses are chosen as to optimize the signal quality of the SPR modes induced at the Au top interface with the probed medium. ${ }^{15,17}$ The calculations thus include aspects such as the propagation distances of the modes, confinement of the fields and the ratio between the diffracted intensities of the signal $v s$. those of the background noise. The choice of periodicity is dictated by the operating energies (near-infrared here) and the aqueous medium of operation. The surface roughness and dielectric constants of all the materials employed have been determined experimentally. ${ }^{17}$

The basic functioning of the QW-SPR device, as detailed in previous communications, ${ }^{9,13,15}$ is described as follows: a broadband light is emitted from the semiconductor substrate in all directions (uncollimated). Given the uncollimated nature of the emissions, all photonic modes supported by the structure are induced concurrently. Amongst those, SPR modes are coupled at the interface between the Au layer and the exposed surface, at the energies and wavevectors corresponding to the SP dispersion relation $E(\mathbf{k})$, such as illustrated in Figure 1a. Trapped at the interface, the SPs are then diffracted by the grating to be observed in the far field (see inset in Figure 2).

Given that coupled SPs travel in all planar directions at the metal surface $(\hat{x}, \hat{y})$, the expected SPR diffraction at a given energy should be circles corresponding to $\mathbf{k}_{/ /}(E)=\left(\left|\mathbf{k}_{x}\right|^{2}+\left|\mathbf{k}_{y}\right|^{2}\right)^{1 / 2}=\mathbf{k}_{\mathrm{SP}}(E)+n\left|\mathbf{k}_{\mathrm{G}}\right|$, with $n$ an integer, $\mathbf{k}_{\mathrm{G}}=2 \pi / P$ in the direction of $\hat{x}$ and $\mathbf{k}_{\mathrm{SP}}(E)$ the resonant condition for the SPR at a given energy. The diameter of the circles a

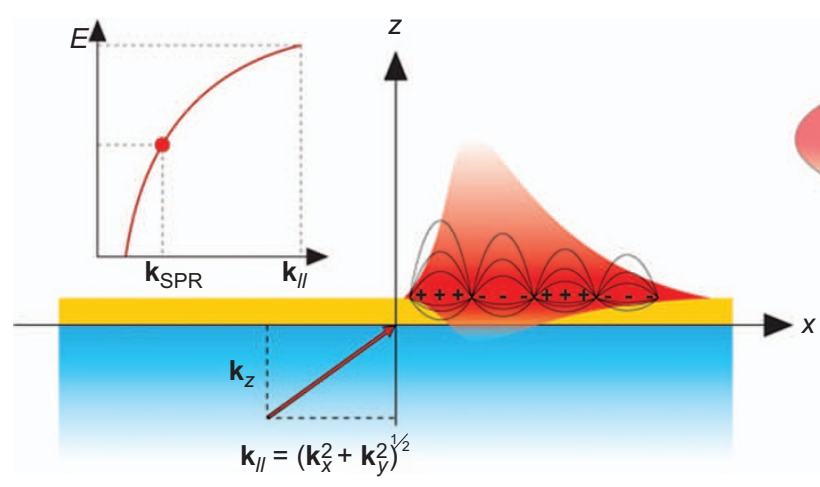

b



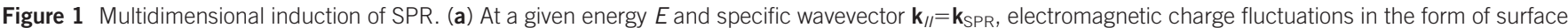

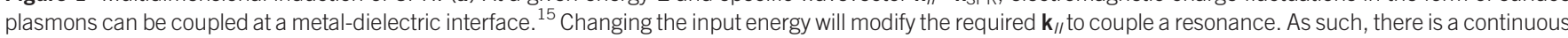

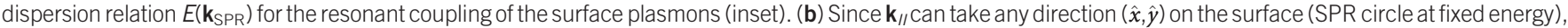

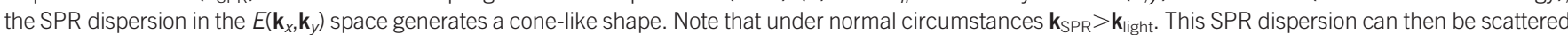
within the light cone by a grating, through the \pm 1 st diffraction order for example, to be measured by a microscope in the far-field. SPR, surface plasmon resonance. 


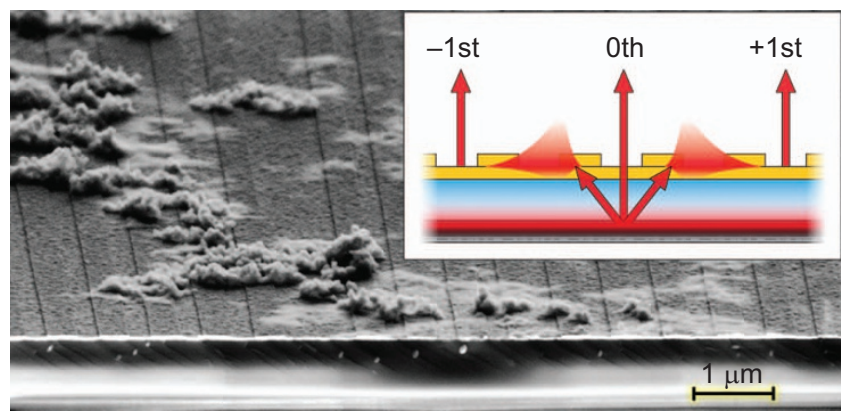

Figure 2 Working principle of the QW-SPR. The SEM image presents a grazing angle view of a device sequentially exposed to solutions of neutravidin, biotinylated polyclonal IAV-H3N2 antibodies and capsids of IAV-H3N2. The Au grating is visible on the surface, under which a $\mathrm{SiO}_{2}$ layer is deposited atop the GaAsAIGaAs QW. Inset: Broadband and uncollimated NIR light is emitted from the QW to couple a continuum of SP modes on the surface, since all the $E\left(\mathbf{k}_{/ /}\right)$are met concurrently. The propagating modes can then diffract, mainly through the \pm 1 st orders, to be measured by the conjugate hyperspectral microscope. IAV, influenza A virus; NIR, near-infrared; QW, quantum well; SEM, scanning electron microscope; SP, surface plasmon; SPR, surface plasmon resonance.

correspond to $\mathbf{k}_{\mathrm{SP}}(E)$ changing with the energy following the specific dispersion of the SPR mode coupled at the moment of observation. Because the dispersion of SPs is close to linear in the visible (VIS) and near-infrared (NIR) for the employed Au-dielectrics, the diffraction of the SPR dispersion resemble cone-like shapes in $E\left(\mathbf{k}_{/ l}\right)$. This is illustrated in Figure 1b, where the SPR dispersion cones are diffracted along the $\mathbf{k}_{x}$-axis within the measurable light cone, and where $E=\hbar c|\mathbf{k}|$. The measured resonance intensities are then dictated by the diffraction efficiencies. We have shown that those can be successfully predicted and measured. ${ }^{12,14,15}$

To study the complete behaviour of the QW-SPR system, a commercial hyperspectral microscope (Photon etc Inc., Montréal, QC, Canada) has been set up to image $\mathbf{k}_{x}-\mathbf{k}_{y}$ directly in the Fourier plane. A schematic idea of this measurement is presented in Figure 3, where the microscope can be employed either for hyperspectral mapping in the $(\hat{x}, \hat{y})$ or wavevector $\left(\mathbf{k}_{x}-\mathbf{k}_{y}\right)$ space, through the adjustment of a kinetic mirror. The collected data are thus cubes of intensities in $I\left(E, \mathbf{k}_{x}, \mathbf{k}_{y}\right)$, yielding the dispersion relation of any induced photonic mode. ${ }^{12,14}$ In the presented case, the dispersion of diffracted SP modes can be studied directly. We also have recently presented a different way of using the same system, which involves the live filming of a conic cross-section of the SPR dispersion relation (elliptical projection) with time resolution down to $1.2 \mathrm{~s} .{ }^{15}$ This method, while not generating the entire $I\left(E, \mathbf{k}_{x}, \mathbf{k}_{y}\right)$ emission map from the light scattering, can still produce a useful section of the dispersion map along the tilted axes of $E-\mathbf{k}_{y}$ and $\mathbf{k}_{x}{ }^{15}$ The SPR shifts can thus be quantified in $\mathbf{k}_{x}$ versus $E$ and $\mathbf{k}_{y}$.

For the dynamic studies of BSA and IAV-H3N2 adsorption, three methods are compared. The first method employs a KretschmannRaether nanoSPR6 system. ${ }^{18}$ Prior to any QW-SPR testing, all the procedures are verified and assessed using this commercial system, whose results serve as standards for comparison with the proposed semiconductor technological platform. The nanoSPR6 is a basic prism setup with a mounted $1.91 \mathrm{eV}(650 \mathrm{~nm})$ laser and automated goniometer with a $10^{-4}$ degree angular resolution. The second method is based on the QW-SPR integrated device, and it is used to collect timeresolved data of the full dispersion relation in $I\left(E, \mathbf{k}_{x}, \mathbf{k}_{y}\right)$. As it will be discussed, the time resolution of this full dispersion method is $360 \mathrm{~s}$. The third method represents the conic methodology approach, ${ }^{15}$ tested under the same conditions, where time-dependent elliptical



Figure 3 Hyperspectral microscope. The MO is focused on the device and the scattered light is collected within the NA. The light bundle can then directly be sent to the VBG imager, which spectrally spreads a real image onto the camera. When activating the kinematic mirror (double arrows), the optical path is elongated and the Fourier plane of the $\mathrm{MO}$ is projected on the camera instead. When observing diffracted SPR, adjusting the VBG allows the direct measurement of the SP $E\left(\mathbf{k}_{\| /}\right)$dispersion relation (Figure 1 ). Given that the $N A<1$ here, only a fraction of the cones are studied. ${ }^{15} \mathrm{MO}$, microscope objective; NA, numerical aperture; SP, surface plasmon; SPR, surface plasmon resonance; VBG, volume Bragg grating.

projections of the SPR dispersion in $I\left(E-\mathbf{k}_{y}, \mathbf{k}_{x}\right)$, are collected from a QW-SPR device. Using this regime, the data collection rate is down to $2.2 \mathrm{~s}$ ( $1 \mathrm{~s}$ of camera exposition and $1.2 \mathrm{~s}$ for the $3.2 \mathrm{Mb}$ frame transfer). No temperature stabilization is employed for any of the investigated methods. For the last two methods, the collected light cone is limited by a $10 \times$ microscope objective $(\mathrm{MO})$, with a 0.3 numerical aperture (NA). In the Fourier plane, the resolution is inversely proportional to the NA. For the applied objective and CCD camera of sensing area of $14.85 \mathrm{~mm} \times 10.00 \mathrm{~mm}$, the acquisition range is typically $\left[2.1 \mu^{-1}\right.$, $\left.2.1 \mu \mathrm{m}^{-1}, 0.3 \mathrm{eV}\right]$ with a resolution of $\left[1.3 \times 10^{-3} \mu \mathrm{m}^{-1}\right.$, $\left.1.3 \times 10^{-3} \mu^{-1}, 1.6 \times 10^{-3} \mathrm{eV}\right]$. Acquisition ranges and resolutions can be adjusted by the selection of a different MO.

It should be noted that multiple methods could be applied to track changes in $I\left(E, \mathbf{k}_{x}, \mathbf{k}_{y}\right)$ as a function of time, which include integrated response, ${ }^{19}$ normalized-difference integrated response ${ }^{20}$ and SPR peak shifting. The first two integrated methods have been found to be relatively unstable for the presented case, as they depend strongly on the time stability of the light source(s) in $I\left(E, \mathbf{k}_{x}, \mathbf{k}_{y}\right)$. For our modest QW light source, characterized by a low luminous flux and no feedback stabilization systems, even post-processing normalization yielded unreliable signal-to-noise ratios (SNRs) while tracking the SPR signal. Therefore, a simple peak tracking in intensity (straightforward neighbors comparison) is used throughout the results section, without any curve fitting. The peak tracking has the disadvantage of only retaining the information about the resonance of the SPs, while discarding all the other photonic changes measured (including the resonance $Q$-factor $)$ in $I\left(E, \mathbf{k}_{x}, \mathbf{k}_{y}\right)$. It is however a well established means of quantifying SPR, independent of the coupling source. ${ }^{4}$

\section{EXPERIMENTAL PROCEDURE}

The first dynamic study involves the evaluation of the QW-SPR sensitivity by measuring the device response to physisorption of BSA. 


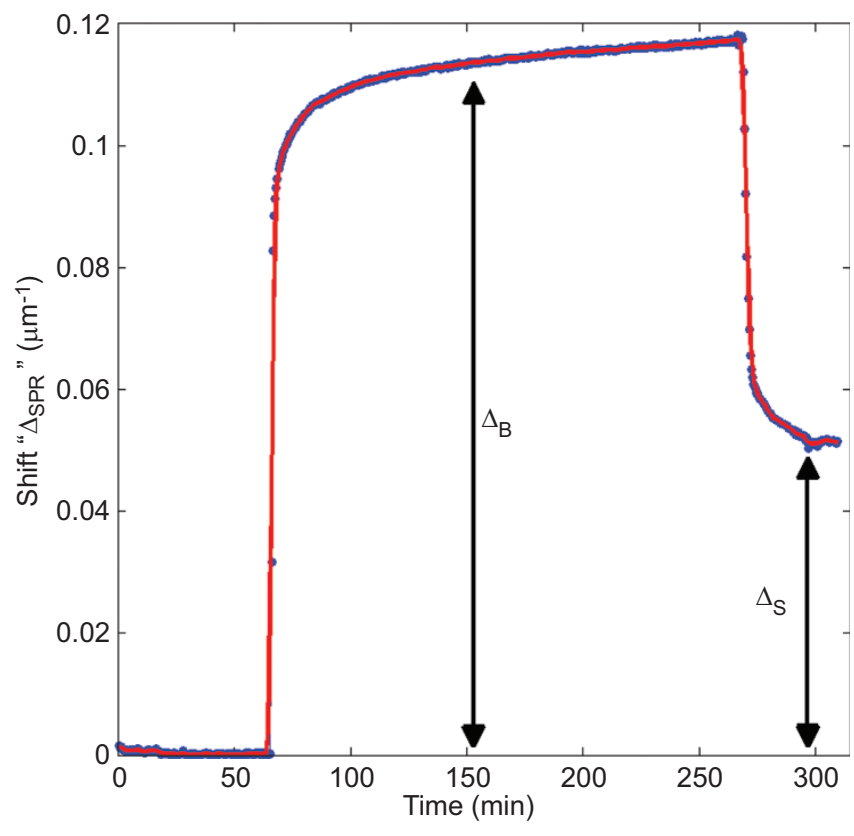

Figure 4 BSA physisorption studied with a commercial system. The well-known physisorption of BSA is employed as a characterization tool for the evaluation of the SNR for all the devices and methods presented. This figure presents the SPR peak shift, in $\mu \mathrm{m}^{-1}$, induced by a $2 \%$ BSA solution. $\Delta_{B}$ is defined as the bulk shift, when the saturation occurs for the investigated solution. $\Delta_{S}$ is defined as the surficial shift, measured after the surface is rinsed by the buffer and only physisorbed molecules contribute to the shift. BSA, bovine serum albumin; SNR, signal to noise ratio; SPR, surface plasmon resonance.

Figure 4 presents an example of the benchmark measurement carried out with the nanoSPR6 system. A phosphate-buffered saline (PBS) solution, with $\mathrm{pH}=7.4$, is first injected into the system and used as background. A 2\% BSA solution in PBS is then introduced in one of the system's channel and left for $180 \mathrm{~min}$. Next, both channels are rinsed with the PBS solution. For BSA, this experiment is repeated five times using the same substrate that is cleaned between experiments to ensure repeatability. The cleaning process consists of a sequence of 5min ultrasonic baths in soap, acetone and isopropyl alcohol, followed by a $20-\mathrm{min} \mathrm{UV}-\mathrm{O}_{3}$ exposure. The prototypes are then put again through the sequence of ultrasonic baths one more time to remove any impurities dislodged by the $\mathrm{UV}-\mathrm{O}_{3}$ cycle.

The SPR shift in the commercial system is given by the difference between the SPR peak positions in the working and reference (exposed to PBS only) channels. The SNR of the method is estimated by smoothing the collected results with a moving average filter: the smoothed curve (full red line in Figure 4), defined as the 'signal' and the 'noise', is the difference between the smoothed signal and the actual data (blue dots in Figure 4). The geometric mean of the ratio between the signal and noise defines the SNR and the geometric standard deviation is used to represent the uncertainty on the estimated SNRs.

It should be noted that there is a difference between absolute hardware resolution and experimental sensitivity, as the later depends on many additional factors other than photonics. This is why the dynamic range of the instrument is also of interest, since it directly represents the experimental sensitivity, i.e., the resulting SPR shift per refractive index change is related to the smallest perturbation detectable. In the presented case, the bulk SPR shift, $\Delta_{\mathrm{B}}$ in Figure 4 is the nominal shift when the substrate is saturated with the $2 \%$ BSA solution, which means the whole evanescent tail of the SPs is exposed to perturbations. This plateau is determined by fitting the physisorption data to an exponential growth-saturation model such as

$$
\Delta_{\mathrm{SPR}}=\Delta_{\mathrm{B}} \cdot \int_{0}^{x^{\prime}} x \cdot e^{-\gamma_{1} \cdot x} \cdot \mathrm{d} x
$$

where $\gamma_{1}$ is a fitted growth rate. The surficial shift, $\Delta_{\mathrm{S}}$ in Figure 4 , is given after the surface is rinsed, when only the adsorbed BSA molecules induce a change. This shift is dictated by the dielectric constant of the molecules, their size and surface coverage, but also by the energy dependent fraction of the SP evanescent field ( $\hat{z}$ in Figure 1) overlapping the adsorbed molecules. The $\Delta_{S}$ is determined by fitting the decay to

$$
\Delta_{\mathrm{SPR}}=\Delta_{\mathrm{B}}-\left(\Delta_{\mathrm{B}}-\Delta_{\mathrm{S}}\right) \cdot \int_{0}^{x^{\prime}} e^{-\gamma_{2} \cdot x} \cdot \mathrm{d} x
$$

Using these established benchmarks, the procedure for BSA physisorption is repeated several times using the QW-SPR architecture presented in Figure 2. Again, a 2\% BSA solution in PBS is injected over the device's surface, left for $180 \mathrm{~min}$ and then rinsed.

In addition to the BSA tests, we investigated the specific adsorption of the IAV (H3N2 strain). This series of measurements is meant to bring the examination of the integrated QW-SPR device one step closer to its targeted set of applications. The goal is to use the biochemistry of $\mathrm{H} 3 \mathrm{~N} 2$ adsorption as a tool to assess the potential limits of the presented structure, and not necessarily to push the detection limit boundaries.

As in the case of physisorbed BSA, immobilization recipes are established using the commercial SPR system. The following procedure is employed for the specific H3N2 immobilization: a $200 \mu \mathrm{g} \mathrm{ml}^{-1}$ neutravidin solution in PBS is injected on the substrate surface, left to physisorb for a 160-min period and rinsed with the PBS buffer. A $100 \mu \mathrm{g} \mathrm{ml}^{-1}$ solution of biotinylated polyclonal IAV-H3N2 antibodies is then injected and left to react with the neutravidin for $160 \mathrm{~min}$. At this stage, the surface is ready to receive a $40 \mu \mathrm{g} \mathrm{ml}^{-1}$ solution containing gamma radiation inactivated IAV. A negative test is carried by first introducing the IAV-H1N1 strain and measuring its surficial shift $\Delta_{\mathrm{S}}$. Then the IAV-H3N2 strain, of identical concentration, is injected and $\Delta_{\mathrm{S}}$ measured for positive identification. For both strains, the solutions are left to react for $60 \mathrm{~min}$ each. Additionally, fluorescein-conjugated biotinylated polyclonal IAV-H3N2 antibodies have been injected to further confirm, by sandwich immunodetection, the selective immobilization of H3N2. Fluorescence microscopy measurements were carried out to assess the macroscopic surface coverage. The experiment is repeated five times using the same substrate (washed between acquisitions using the method described above). Supplementary Fig. S1 presents an example for the dynamic of selective H3N2 immobilization using the commercial nanoSPR6 system.

\section{RESULTS}

For the typical nanoSPR6 BSA physisorption results presented in Figure 4 , the $\mathrm{SNR}=501 \pm 13$. Again, this result is the geometric mean and standard deviation of the ratio between the smoothed curve (signal) and the subtracted raw data (noise) in Figure 4. For a typical BSA nanoSPR6 experiment, $\Delta_{\mathrm{B}}=1081 \times 10^{-4} \pm 3 \times 10^{-4} \mu \mathrm{m}^{-1}$ and after the PBS rinse, $\Delta_{\mathrm{S}}=519 \times 10^{-4} \pm 3 \times 10^{-4} \mu \mathrm{m}^{-1}$ is found by fitting with the above-mentioned decay model. 
The specific immobilization of inactivated IAV-H3N2 on the commercial SPR platform is presented in Supplementary Fig. S1. The SNR for the system is comparable to the simpler BSA experiments. What is of interest in this case are the $\Delta_{\mathrm{SPR}}$ for the influenza viruses. The negative test using IAV-H1N1 yielded $\Delta_{\mathrm{B}}(\mathrm{H} 1 \mathrm{~N} 1)=55 \times$ $10^{-4} \pm 9 \times 10^{-4} \mu \mathrm{m}^{-1}$ and $\Delta_{\mathrm{S}}(\mathrm{H} 1 \mathrm{~N} 1)=-10 \times 10^{-4} \pm 10 \times 10^{-4} \mu \mathrm{m}^{-1}$. The positive test, performed by injecting the same concentration of IAV-H3N2, gave $\Delta_{\mathrm{B}}(\mathrm{H} 3 \mathrm{~N} 2)=124 \times 10^{-4} \pm 4 \times 10^{-4} \mu^{-1}$ and $\Delta_{\mathrm{S}}(\mathrm{H} 3 \mathrm{~N} 2)=92 \times 10^{-4} \pm 7 \times 10^{-4} \mu \mathrm{m}^{-1}$ for the commercial system. These results infer, as expected, a distinct preference for the H3N2 strain over the H1N1. The $\Delta_{\mathrm{S}}(\mathrm{H} 1 \mathrm{~N} 1) \neq 0$ can be explained by the use of IAV-H3N2 polyclonal antibodies of broader affinity than their monoclonal counterpart and by an imperfect surface coverage of the physisorbed neutravidin complex, leaving Au regions potentially exposed to H1N1 capsids. Fluorescence imaging over the surfaces exposed to H3N2 fluorescein-conjugated antibodies also showed the positive immobilization of the inactivated virus. Scanning electron microscope imaging showed an average surface coverage of 5 capsids $\mu \mathrm{m}^{-2}$.

These results demonstrate reliable immobilization dynamics and comparison benchmarks for both the BSA molecule and IAV-H3N2 virus, on which the evaluation of the QW-SPR platform can be established. The procedures are then transferred to the QW-SPR device and inspected using the two different hyperspectral acquisition methods.

The dynamics of the full SP dispersion relation shifts is first observed. The BSA physisorption procedure is repeated over the QW-SPR and Figure 5a presents the response from a single hyperspectral cube at a given time, where only the SPR peaks (surfaces in 3D) are presented for clarity. In reality, a complete $I\left(E, \mathbf{k}_{y}, \mathbf{k}_{x}\right)$ dataset is collected as a function of time. Two diffracted SP surfaces are observable with the $10 \times \mathrm{MO}$, corresponding to the \pm 1 st diffraction orders. These surfaces move in time as BSA molecules are adsorbed on the Au substrate. A dynamic version of Figure 5a, observed during injection of the BSA solution, is available in Supplementary Movie 1. The distances, in $\mathbf{k}_{x}$, between the two surfaces can then be projected as a function of the emitted energy $\left.\Delta_{\mathrm{SPR}}(E)\right|_{k y}$, as illustrated in Figure $5 \mathrm{~b}$. A different cross-section of the peak shift $\left.\Delta_{\mathrm{SPR}}\left(\mathbf{k}_{y}\right)\right|_{E}$ is also presented in Figure $5 c$. The SPR is not necessarily homogeneous as a function of $E$ or $\mathbf{k}_{y}$, as it depends on the spectral response of the biochemicals and photonic structures present (such as the grating). For a comparable dynamic study with the commercial system's benchmarks, the SPR shifts can be projected into a single total dispersion shift versus time, as shown in Figure 5d. Here, the cumulative shift is the distance between the two SPR peak surfaces, given by $\left.\Delta_{\mathrm{SPR}}\right|_{\text {Total }}=$ $\left\{\left.\sum \Delta_{\mathrm{SPR}}\left(\mathbf{k}_{y}\right)\right|_{E}{ }^{2}+\left.\sum \Delta_{\mathrm{SPR}}(E)\right|_{k y}{ }^{2}\right\}^{1 / 2}$. As in the benchmark experiment, it is possible to estimate the resulting SNR to be $655 \pm 16$, with $\Delta_{\mathrm{B}}=233 \times 10^{-2} \pm 3 \times 10^{-2} \mu \mathrm{m}^{-1}$ and $\Delta_{\mathrm{S}}=114 \times 10^{-2} \pm 3 \times 10^{-2} \mu \mathrm{m}^{-1}$. Subsequently, the IAV-H3N2-specific immobilization procedure is performed on the QW-SPR. A static cube of the acquisition, presented in Supplementary Fig. S2a, is similar to that in Figure 5a, and a movie of the dispersion change in time is available in the provided Supplementary Movie 2. Cross-sections of the dispersion in $\left.\Delta_{\mathrm{SPR}}(E)\right|_{k y}$ and $\left.\Delta_{\mathrm{SPR}}\left(\mathbf{k}_{y}\right)\right|_{E}$, are also shown in Supplementary Fig. S2b and S2c. The total SPR shift is presented in Supplementary Fig. S2d. The SNR is consistent with the previous BSA experiments. The relevant shifts for the specific immobilization of IAV-H3N2 are $\Delta_{\mathrm{B}}=66 \times 10^{-2} \pm 1 \times 10^{-2} \mu \mathrm{m}^{-1}$ and $\Delta_{\mathrm{S}}=57 \times 10^{-2} \pm 2 \times 10^{-2} \mu \mathrm{m}^{-1}$.

We have also investigated the conic mode of SPR data collection as an approach towards the rapid data collection with the hyperspectral imaging technology. Figure 6 a shows a single frame $(2.2 \mathrm{~s}$ collection time) result obtained with this approach during physisorption of BSA on the surface of the QW-SPR device. Two diffracted SP modes are clearly visible and their position traceable in time. The time-dependent BSA adsorption procedure, represented by $\Delta_{\mathrm{SPR}}$ in $\mathbf{k}_{x}$ as a function of $E-\mathbf{k}_{y}$ for each frame, is presented in Figure $6 \mathrm{~b}$. As in the full-time scan SPR method, the cumulative shift is presented in Figure $6 \mathrm{c}$, where $\left.\Delta_{\mathrm{SPR}}\right|_{\text {Total }}=\left\{\sum \Delta_{\mathrm{SPR}}\left(E-\mathbf{k}_{y}\right)^{2}\right\}^{1 / 2}$. For the conic method, the $\mathrm{SNR}=1831 \pm 12, \Delta_{\mathrm{B}}=7569 \times 10^{-4} \pm 7 \times 10^{-4} \mu \mathrm{m}^{-1}$ and $\Delta_{\mathrm{S}}=7140 \times$ $10^{-4} \pm 60 \times 10^{-4} \mu \mathrm{m}^{-1}$. Subsequently, the specific immobilization of IAV-H3N2 is carried out on a QW-SPR device, whose SPR fluctuations are now monitored using the conic method. Supplementary Fig. S3a shows the SPR shift, $\Delta_{\mathrm{SPR}}\left(E-\mathbf{k}_{y}\right)$ as a function of time. The summation of the shifts in a conventional SPR signal is presented in Supplementary Fig. S3b, where $\Delta_{\mathrm{B}}=35 \times 10^{-3} \pm 1 \times 10^{-3} \mu^{-1}$ and $\Delta_{\mathrm{S}}=33 \times 10^{-3} \pm 1 \times 10^{-3} \mu_{\mathrm{m}}^{-1}$ are induced by specific interaction of the inactivated H3N2 virus with the QW-SPR. Supplementary Tab. S2 summarizes all the SPR shifts for all the presented methods.

\section{DISCUSSION}

When comparing the SNR of the SPR signal generated by the QW-SPR architecture with that of the nanoSPR6 commercial system, we find the former generates slightly more stable datasets. The investigated QW-SPR architecture is examined in the NIR region of emission, which results in a larger evanescent field in the $\hat{z}$ direction than that corresponding to the VIS emission of the nanoSPR6 system. Indeed, for the QW-SPR system, at $1.42 \mathrm{eV}$ the SP E-field reaches $e^{-1}$ after $322 \mathrm{~nm}$ in $\hat{z}$, while the nanoSPR6 SP, at $1.91 \mathrm{eV}$, reaches the same intensity ratio after only $199 \mathrm{~nm}$ (in PBS). The larger depth of the evanescent field technically lowers the SPR sensitivity for small molecules such as BSA. ${ }^{4}$ This is one factor contributing to the stabilization of the QW-SPR signal, at the cost of sensitivity. Despite that fact, the bulk $\left(\Delta_{\mathrm{B}}\right)$ and surficial $\left(\Delta_{\mathrm{S}}\right)$ shifts, for both the full and conic hyperspectral collection methods, are much larger than their KretschmannRaether prism counterparts. This is attributed to two factors. Firstly, the grating offers a small increase in surface area due to corrugation (6\%) and an enhanced efficiency of the SPR response for adsorbed molecules on nanopatterned surfaces. ${ }^{21}$ Secondly, the continuous surface coupling of the SPR over a wide range of $E$ and $\mathbf{k}_{/ /}$results in the cumulative shifts over the collected dispersions which are greater than the potential shift over single $E$ or $\mathbf{k}_{/ /}$values.

However, a comparison between Figure 4 and $5 \mathrm{~d}$ shows that the number of collected data points is quite smaller for the case of the full dispersion approach. This is mainly due to the difference in the luminous flux of the pump signals: the benchmark nanoSPR6 operates with a VIS laser, while the SPR data in Figure 5 are induced by the luminescence of a single quantum well, purposefully spread over a wide range of $E$ and $\mathbf{k}_{\| /}$. It takes $360 \mathrm{~s}$ to collect each full hyperspectral cube over a range of $\left[2.1 \mathrm{~mm}^{-1}, 2.1 \mathrm{~m}^{-1}, 0.3 \mathrm{eV}\right]$ with a resolution of $\left[3.9 \times 10^{-3} \mu \mathrm{m}^{-1}, 3.9 \times 10^{-3} \mu \mathrm{m}^{-1}, 3.2 \times 10^{-3} \mathrm{eV}\right] .^{15}$ This scale of acquisition time could be sufficient for some slower biochemical analyses, but would present problems in the case of faster reactions. This relatively large time resolution also increases the uncertainty over the measured shifts, given the relatively small dataset.

While the SPR data collected with the conic cross-section method are less complete than those obtained with the full dispersion mapping, ${ }^{15}$ they still offer satisfactory results with a relatively high SNR. In the case presented in Figure 6, each frame that is recorded every $2.2 \mathrm{~s}$ has a resolution of $\left[1.3 \times 10^{-3} \mu \mathrm{m}^{-1}, 1.4 \times 10^{-3} \mu \mathrm{m}^{-1}\right]$. The acquisition time is mostly limited by the integration time of the QW luminescent emission. As in the full hyperspectral method, a stronger intensity source would decrease the acquisition time and increase 

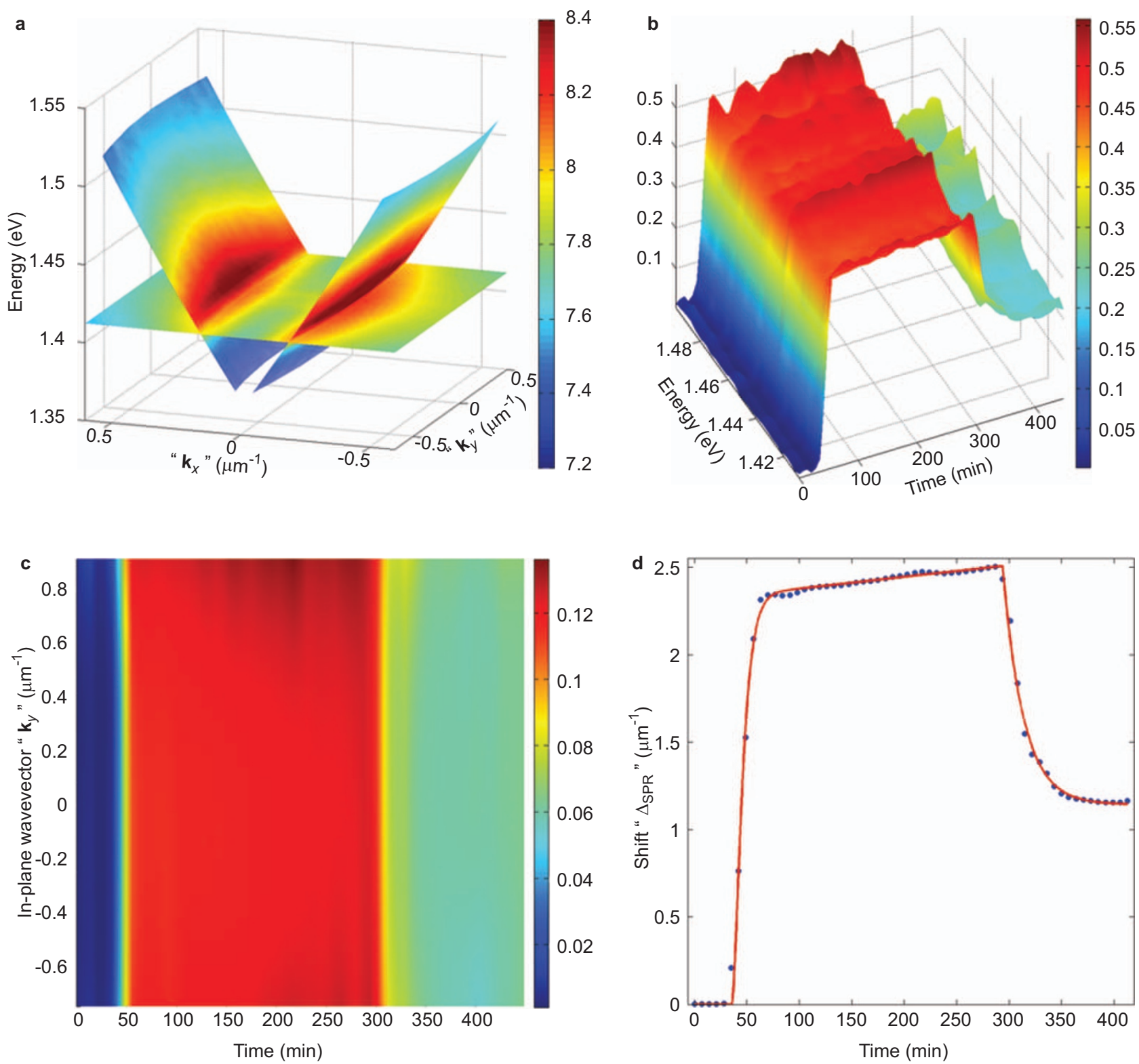

Figure 5 BSA physisorption on a QW-SPR device. (a) The dispersion relation in $E\left(\mathbf{k}_{/ /}\right)$of the diffracted SPs is recorded as a hyperspectral cube at a given time. ${ }^{15}$ The distance between the two surfaces is measured, in time, to assess the changes induced by the BSA molecule. The intensity is given in a logarithmic scale. (b) The SPR shift $\left.\Delta_{\mathrm{SPR}}(E)\right|_{\mathrm{ky}}$, in $\mu \mathrm{m}^{-1}$, as a function of energy and time, as the $2 \%$ solution is injected. (c) The same data collection this time distributed over the in-plane wavevector $\mathbf{k}_{y}$ as a function of time. The color scale represents the SPR shift, $\left.\Delta_{S P R}\left(\mathbf{k}_{y}\right)\right|_{E}$, in $\mu \mathrm{m}^{-1}$. (d) The same dataset compressed into a 2D graph comparable to Figure 4, where the SNR, bulk shift $\left(\Delta_{\mathrm{B}}\right)$ and surficial shift $\left(\Delta_{\mathrm{S}}\right)$ are measured. BSA, bovine serum albumin; QW, quantum well; SNR, signal-to-noise ratio; SP, surface plasmon; SPR, surface plasmon resonance.

the SNR of the method. Although smaller than the full dispersion approach, the dynamic range of the total SPR shift is nearly one order of magnitude larger than the classical Kretschmann-Raether approach, attributed to the recorded cumulative shifts in $E-\mathbf{k}_{y}$. Supplementary Tab. S1 summarizes the various capabilities of the methods presented.

The mass coverage for the $0.3 \mu \mathrm{M}$ BSA solution on Au presented here is estimated to be $994.9 \mathrm{pg} \mathrm{mm}^{-2}$, which is a mid-range value when compared with the literature. ${ }^{22,23}$ The bulk $\left(\Delta_{\mathrm{B}}\right)$ refractive index change induced by $0.3 \mu \mathrm{M}$ BSA in PBS buffer is 0.0037 RIU for both the VIS and NIR. ${ }^{24-26}$ After rinsing, the $994.9 \mathrm{pg} \mathrm{mm}^{-2}$ BSA coverage induces an effective refractive index shift of the SPR modes of
$\Delta n_{\text {eff }}=16.75 \times 10^{-4}$ RIU for the $1.91 \mathrm{eV}$ SPs and $\Delta n_{\text {eff }}=10.39 \times 10^{-4}$ RIU at $1.42 \mathrm{eV}$. Therefore, from the SPR shifts and SNR, summarized in Supplementary Tab. S2, it is possible to estimate the minimum refractive index change measurable by each method. In bulk situations, when the SP modes are completely covered by a substance, the nanoSPR6 method yields a $1.0 \times 10^{-5}$ RIU resolution, the full dispersion mapping $4.8 \times 10^{-5}$ and $3.4 \times 10^{-6}$ RIU for the conic approach. Under conditions of surficial detection, the resolutions for the three methods are $9.7 \times 10^{-6}, 2.7 \times 10^{-5}$ and $1.5 \times 10^{-6} \mathrm{RIU}$ for the nanoSPR6, NIR full dispersion mapping and NIR conic mapping, respectively. The surprising performance of the conic approach is explained by an optimal tradeoff between an attractive dynamic 

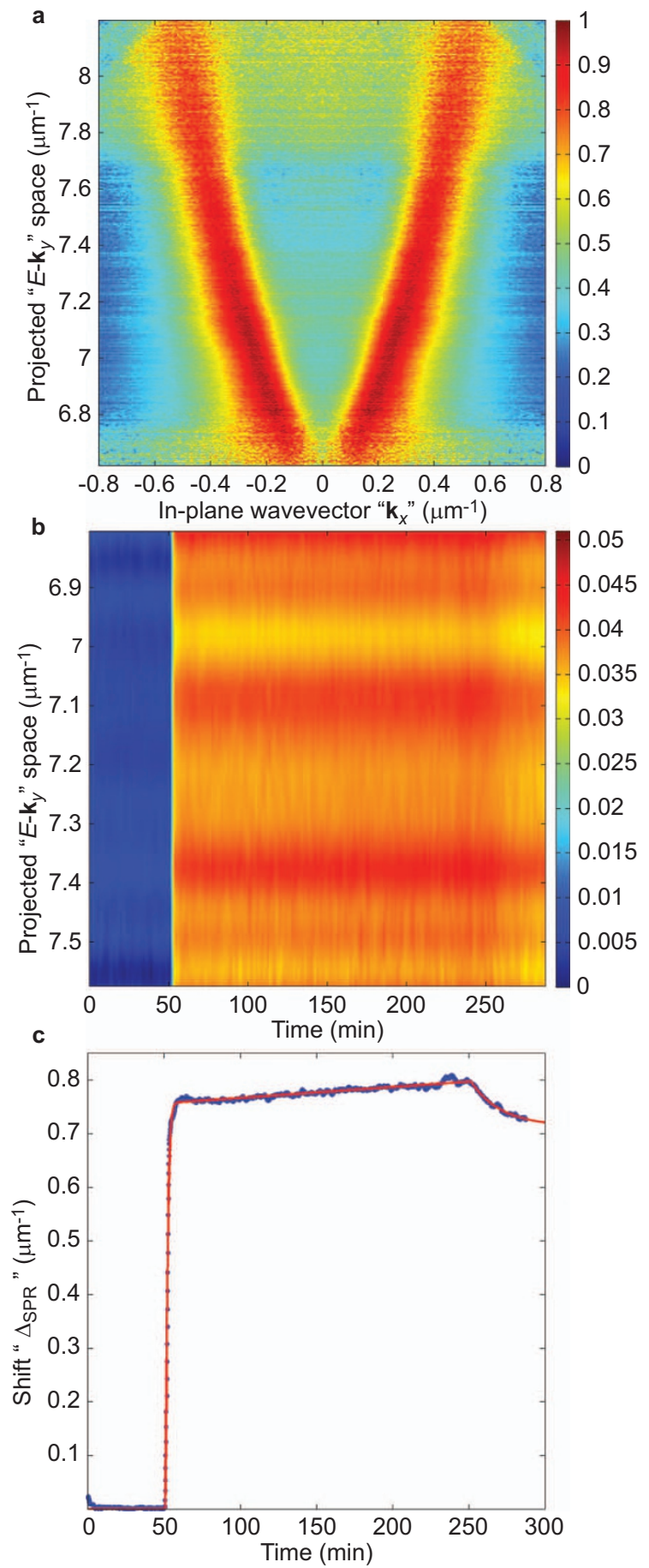

Figure 6 Conic measurement of BSA physisorption on the QW-SPR device. (a) The dispersion relation of the SPR is projected on a skewed set of axes $I\left(E-\mathbf{k}_{y}, \mathbf{k}_{x}\right) \cdot{ }^{15}$ The image reveals the projection of two ellipses, obtained from the projection of the SPR dispersion on the $E-\mathbf{k}_{y}$ space. Measuring the distance in $\mathbf{k}_{x}$ between the two ellipses provides direct information on the SPR conditions. In this case, the quality of the signal as a function of $E-\mathbf{k}_{y}$ depends on the luminescence intensity of the QW. (b) $\Delta_{\mathrm{SPR}}$ as a function of $E-\mathbf{k}_{y}$ and time, as the BSA solution is injected over the device and rinsed. The periodic modulations in $E-\mathbf{k}_{y}$ are attributed to the interactions of the SPs with the grating as predicted in the literature. ${ }^{15,16}$ (c) Cumulative SPR shift in time for the BSA physisorption, where the SNR, bulk shift $\left(\Delta_{B}\right)$ and surficial shift $\left(\Delta_{\mathrm{S}}\right)$ are measured. BSA, bovine serum albumin; QW, quantum well; SNR, signalto-noise ratio; SP, surface plasmon; SPR, surface plasmon resonance. range and a stable signal, with many points, in time (SNR). It is important to note that the resulting QW-SPR sensitivities are found to be comparable to the best reported in the SPR literature. ${ }^{27,28}$

The specific immobilization and detection of the IAV-H3N2 has demonstrated the potential to use the QW-SPR device as an active biosensor. The challenges in detecting IAV in solution with SPR lies in both the chemistry involved and the small RIU changes induced. Indeed, the porous IAV shell is permeable to the PBS buffer: only the genetic material and the proteins forming the capsid contribute to the refractive index change for a given surface coverage. Specific shifts induced by the inactivated virus were measured by all the presented methods. As in the BSA experiment, the dynamic range of the QW-SPR has been found superior to the commercial system, despite the low-power light source. This phenomenon is attributed to the cumulative effect of the shifts oven the entire $E\left(\mathbf{k}_{\|}\right)$dispersion relation monitored within the NA of the $10 \times \mathrm{MO}$. The total shift for the conic method is, however, smaller than expected, when comparing with the proportions of $\Delta_{\mathrm{S}}$ of the IAV-H3N2 experiment $\left(57 \times 10^{-2}\right.$ and $33 \times 10^{-3}$ for the full hyperspectral and conic methods respectively) to the $\Delta_{\mathrm{S}}$ in the BSA experiments $\left(114 \times 10^{-2}\right.$ and $714 \times 10^{-3}$ for the same order). This could be explained by the relatively low surface coverage ( 5 viruses $\mu \mathrm{m}^{-2}$ ) and small size of the IAV-H3N2. It is, however, likely to be attributed to the QWSPR prototype device wearing down, as in the last sequence of experiments, it had been exposed to over $55 \mathrm{~h}$ of detection in addition to four washing sequences. Scanning electron microscope imaging has shown signs of mechanical deterioration on the $\mathrm{Au}$ layer. Despite this fact, the SPR shifts of the QW-SPR, when observed under the conic approach, are still higher than those observed with the nanoSPR6 system. While higher luminous flux from the QW source would enable higher resolution and yield better SNR, the $2.2 \mathrm{~s}$ collection time should be considered attractive not only for biosensing applications of QW-SPR devices, but also for the dynamic study of other scattering events.

\section{CONCLUSIONS}

Current SPR technological platforms are attractive tools employed for a wide range of applications where surface modifications are involved, including most kinetic and thermodynamic analyses of ligand and receptor interactions ${ }^{5}$ that can be employed for biodetection. Introducing an SPR transducer method into a $\mu$ TAS system would enable a dissemination of this characterization method into other fields of applications that currently cannot afford the space, capital or trained personnel to carry out complete experiments. An efficient way to integrate SPR and $\mu$ TAS systems is through the use of existing semiconductor technology, where an integrated light source and transducer can be part of a single nanosystem embedded within a relevant microelectronic circuit. In order to achieve such miniaturization, the concept of SPR prism coupling is replaced by a monolithic architecture.

For that purpose, the general case of broadband and uncollimated semiconductor light-emitters has been studied. We have discussed how to predict the entire nanophotonic behavior of the resulting device and how to use a modified hyperspectral microscope to directly measure the Fourier plane of the SPs scattered by the architecture. This resulted in a detailed description of the dispersion relation of the EMfield at the QW-SPR surface. The advantage of the presented innovation is threefold:

1. A functional SPR transducer integrated with a semiconductor light source that results in an unmatched scaling of the device. Other 
lab-on-chip technologies miniaturizing the SPR method are limited either by the physical dimensions of the prism used for near-field coupling, or by the nature of the light sources, imposing macroscopic limitations on the SPR device geometry. Here the light source-transducer distance has been brought to the ultimate minimum. An understanding of the integration with the semiconductor substrate opens a wide range of new applications that go beyond the field of biosensing. It also makes it possible to interface this platform with traditional microelectronics.

2. Our approach is not limited by the results of one-dimensional plasmonic dispersion relation. In contrast with others, we take advantage of all the SPR energies and all the wavevectors. The analysis of the measurements obtained with this innovative approach is accomplished through the conjugate field of a microscope and, as a consequence of the simultaneous inclusion of a wide spectrum of energies and wavevectors, offers sensitivities that exceed those achievable with conventional SPR systems.

3. We have introduced SPR tracking through microscopy that is a more practical and affordable approach in comparison to conventional methods of monitoring SPR signals.

Dynamic studies of BSA and IAV (H3N2) adsorptions were presented in order to demonstrate the sensitivity and potential dynamic range of an integrated QW-SPR architecture. When comparing the measured SNR of the SPR shift between the monolithic architecture and the commercial Kretschmann-Raether setup, we found the former to be more stable in time and to produce larger shifts for any given reactions. This was attributed to the cumulative effect of the dispersion relation shifts over a wider range of energies and wavevectors. The full dispersion mapping of the SPR has, however, a drawback related to the excessive acquisition time (in the order of minutes per SPR cube of data). This problem was addressed by introducing a novel approach of quasi-hyperspectral imaging, where a conic section of the SPR dispersion (ellipses) is directly projected on a camera. This method yielded high sensitivity, down to $1.5 \times 10^{-6} \mathrm{RIU}$, due to a good balance between SNR and dynamic range. Using a monolithically integrated semiconductor SPR sensor operating in the conic approach, we have demonstrated the feasibility of the method for detecting specific strains of the IAV with $2.2 \mathrm{~s}$ time resolution.

The presented work highlights the introduction of high-quality SPR measurements for studying biomolecular and viral dynamics using a simple semiconductor nanodevice in conjugation with a microscope. While this does not represent a wholly integrated $\mu$ TAS, this approach already represents a more affordable method to carry biochemical analyses to most physicians and biomedical researchers. The understanding and application of uncollimated and broadband SPR results are also paving the way for the application of diffuse SPR phenomena in other fields of nanophotonics.

\section{ACKNOWLEDGMENTS}

The authors acknowledge the financial contribution from the Natural Science and Engineering Research Council of Canada (NSERC Strategic grant STPGP 350501-07), the Canada Research Chair in Quantum Semiconductors Program and the Vanier Scholarship CGS program. The authors also want to thank Ėric Frost, Mohamed Walid Hassen and Dominic Carrier for helpful discussions.
1 Marescaux J, Leroy J, Gagner M, Rubino F, Mutter D et al. Transatlantic robot-assisted telesurgery. Nature 2001; 413: 379-380.

2 Tseng D, Mudanyali O, Oztoprak C, Isikman SO, Sencan I et al. Lensfree microscopy on a cellphone. Lab Chip 2010; 10: 1787-1792.

3 Lee SJ, Lee SY. Micro total analysis system (mu-TAS) in biotechnology. App/ Microbiol Biotechnol 2004; 64: 289-299.

4 Raether $\mathrm{H}$. Surface-plasmons on smooth and rough surfaces and on gratings. Springer Tracts Mod Phys 1988; 111: 1-133.

5 Schasfoort RB, Tudos AJ. Handbook of Surface Plasmon Resonance. Cambridge: Royal Society of Chemistry, 2008.

6 Kabashin AV, Evans P, Pastkovsky S, Hendren W, Wurtz GA et al. Plasmonic nanorod metamaterials for biosensing. Nat Mater 2009; 8: 867-871.

7 de Bruijn HE, Kooyman RP, Greve J. Surface plasmon resonance microscopy: improvement of the resolution by rotation of the object. Appl Opt 1993; 32: 2426-2430.

8 Rothenhausler B, Knoll W. Surface-plasmon microscopy. Nature 1988; 332: 615617.

9 Lepage D, Jiménez A, Carrier D, Beauvais J, Dubowski JJ. Conic hyperspectral dispersion mapping applied to semiconductor plasmonics. Light Sci App/ 2012; 1: e28.

10 Duplan V, Miron Y, Frost E, Grandbois M, Dubowski JJ. Specific immobilization of influenza A virus on GaAs (001) surface. J Biomed Opt 2009; 14: 054042-054046.

11 Hassen WM, Duplan V, Frost E, Dubowski JJ. Quantitation of influenza A virus in the presence of extraneous protein using electrochemical impedance spectroscopy. Electrochim Acta 2011; 56: 8325-8328.

12 Romanato F, Lee KH, Kang HK, Ruffato G, Wong CC. Sensitivity enhancement in grating coupled surface plasmon resonance by azimuthal control. Opt Express 2009; 17: 12145-12154.

13 Lepage D, Jiménez A, Carrier D, Beauvais J, Dubowski JJ. Hyperspectral imaging of diffracted surface plasmons. Opt Express 2010; 18: 27327-27335.

14 Glytsis EN, Gaylord TK. Rigorous 3-D coupled wave diffraction analysis of multiple superposed gratings in anisotropic media. Appl Opt 1989; 28: 2401-2421.

15 Lepage D, Dubowski JJ. Surface plasmon effects induced by uncollimated emission of semiconductor microstructures. Opt Express 2009; 17: 10411-10418.

16 Lepage D, Carrier D, Jimenez A, Beauvais J, Dubowski JJ. Plasmonic propagations distances for interferometric surface plasmon resonance biosensing. Nanoscale Res Lett $2011 ; 6: 388$.

17 Jimenez A, Lepage D, Beauvais J, Dubowski JJ. Study of surface morphology and refractive index of dielectric and metallic films used for the fabrication of monolithically integrated surface plasmon resonance biosensing devices. Microelectron Eng 2012; 93: 91-94.

18 NanoSPR. Available at http://www.nanospr.com, 2012

19 Das M, Hohertz D, Nirvan R, Brolo AG, Kavanagh KL et al. Improved performance of nanohole surface plasmon resonance sensors by the integrated response method. IEEE Photon J 2011; 3: 441-449.

20 Stewart ME, Yao JM, Maria J, Gray SK, Rogers JA et al. Multispectral thin film biosensing and quantitative imaging using 3D plasmonic crystals. Anal Chem 2009; 81: 5980-5989.

21 Hoa XD, Kirk AG, Tabrizian M. Enhanced SPR response from patterned immobilization of surface bioreceptors on nano-gratings. Biosensors Bioelectron 2009; 24: 3043-3048.

22 Azzam RM, Rigby PG, Krueger JA. Kinetics of protein adsorption and immunological reactions at a liquid/solid interface by ellipsometry. Phys Med Biol 1977; 22: 422.

23 Tencer M, Charbonneau R, Lahoud N, Berini P. AFM study of BSA adlayers on Au stripes. App/ Surf Sci 2007; 253: 9209-9214.

24 Akimoto T, Sasaki S, Ikebukuro K, Karube I. Refractive-index and thickness sensitivity in surface plasmon resonance spectroscopy. Appl Opt 1999; 38: 4058-4064.

25 Barer R, Tkaczyk S. Refractive index of concentrated protein solutions. Nature 1954; 173: 821-822.

26 Ioseliani OR. Focus on Eye Research. New York: Nova Science Publishers, 2006.

27 Hoa XD, Kirk AG, Tabrizian M. Towards integrated and sensitive surface plasmon resonance biosensors: a review of recent progress. Biosensors Bioelectron 2007; 23: $151-160$.

28 Huang Y, Ho HP, Kong SK, Kabashin AV. Phase-sensitive surface plasmon resonance biosensors: methodology, instrumentation and applications. Ann Phys (Berlin) 2012; 524: 637-662.

This work is licensed under a Creative Commons Attribution-NonCommercial-NoDerivative Works 3.0 Unported License. To view a copy of this license, visit http:// creativecommons.org/licenses/by-nc-nd/3.0 